\title{
Glucocorticoid induced hyperglycaemia
}

\author{
M I Weerakkody ${ }^{1}$, N P Somasundaram ${ }^{2}$ \\ Sri Lanka Journal of Diabetes, Endocrinology and Metabolism 2012; 2: 25-27
}

\begin{abstract}
Modern medicine has discovered numerous benefits of supra-physiological doses of glucocorticoids, both as immunosuppressive as well as anti inflammatory agents. Hyperglycaemia is one important side effect of steroids, with a number of unresolved issues on its diagnosis and management. This article describes important points regarding the pathophysiology and diagnosis of steroid induced hyperglycaemia. It also discusses a practical approach to its management.
\end{abstract}

\section{Introduction}

The use of glucocorticoids as anti inflammatory and immunosuppressive agents are on the rise, with a parallel increase of the adverse effects of prolonged and supraphysiological dose medications. Glucocorticoids exacerbate hyperglycaemia in almost all patients with diabetes mellitus, but it is also responsible for the development of diabetes mellitus in previously normoglycaemic individuals.

In a nested case control study using the Health Improvement Network in the United Kingdom, oral glucocorticoid therapy was associated with an odds ratio of 1.36 for the development of new onset diabetes (1). Other studies have recorded odds ratios for new onset diabetes due to exogenous steoroids between 1.7 to 2.31 (2). In all these studies the total glucocorticoid dose and the duration of therapy were strong predictors of the induction of diabetes. This is substantiated by evidence that patients with decreased insulin secretory reserve are more likely to develop diabetes (3). Other risk factors are older age and higher body mass index, both of which are known risk factors for the development of insulin resistance and type 2 diabetes mellitus.

The occurrence of hyperglycaemia with glucocorticoids may remain undetected in a proportion of patients as glucocorticoids are more likely to increase post prandial blood glucose than fasting blood glucose. In a study among patients with primary renal disease treated with prednisolone, $40 \%$ developed 2 hour post prandial blood glucose values exceeding $200 \mathrm{mg} / \mathrm{dl}$ but with normal fasting blood glucose (4). In a cohort of patients with various neurological diseases receiving prednisolone, corticosteroid related diabetes developed in 50\%. These patients also had a similar pattern of abnormally high post prandial blood glucose with normal fasting values (5).

\section{Mechanisms of glucocorticoid induced hyperglycaemia}

The predominant mechanism responsible for glucose intolerance associated with glucocorticoid therapy is reduced insulin sensitivity. This impairment in insulin action has been demonstrated in both liver and skeletal muscle. The ability to compensate for this decrease in insulin sensitivity, by an increase in insulin secretion, determines the extent of the rise in plasma glucose with glucocorticoids (6). A reduction in insulin secretory capacity has also been observed at higher doses of corticosteroids (7). Calcineurin inhibitors such as cyclosporine and tacrolimus are known to induce glucose intolerance through a direct effect on insulin synthesis and release. Toxic levels of the drugs have also been shown to induce islet cell apoptosis (8). Due to the dual effects of glucocorticoids and calcineurin inhibitors, the incidence of new onset diabetes in patients undergoing organ transplantation is quite high; the incidence ranging from $10 \%$ to $50 \%$ being reported (2). In addition hyperglycaemia induced by glucocorticoids is known to be associated with reduced glucose transporter 2 (GLUT2) receptor expression and decreased glucose transport of glucose into pancreatic beta cells (9).

The pharmacokinetic properties of most glucocorticoids are short lasting; but their pharmacodynamic properties with respect to glucose tolerance are prolonged, as there are genomic effects of the drugs on mediating gluconeogenesis and peripheral insulin sensitivity. Although the plasma half life of prednisolone and dexamethasone are about 2.5 hours, in a recent study insulin concentrations were significantly elevated even after 20 hours of administration of dexamethasone to normal volunteers suggesting that the hyperglycaemic effect is longlasting (10). 


\section{Management of glucocorticoid induced hyperglycaemia}

The detection of glucocorticoid induced hyperglycaemia requires frequent monitoring of blood glucose levels; self monitoring of blood glucose would therefore play an important role. As the typical pattern of hyperglycaemia with steroids is minimally elevated fasting blood glucose levels with exaggerated post prandial hyperglycaemia, measurement of post prandial blood glucose should be the key screening tool (11).

Treatment of glucocorticoid induced hyperglycaemia, even in the short term, is important. Studies show that even short term post prandial hyperglycaemia is associated with acute inflammation and endothelial dysfunction in patients with and without type 2 diabetes mellitus (12). Reduction of fluctuations in glucose levels with administration of insulin decreases these defects. Although evidence from randomised trials testing this hypothesis in glucocorticoid treated patients is lacking, this phenomenon could be extrapolated as a likely occurrence with glucocorticoid treatment. Healthy dietary practices with avoidance of overeating and the consumption of concentrated simple carbohydrates, regular exercise and regular monitoring of blood glucose levels play an important role in the prevention and treatment of steroid induced hyperglycaemia.

All currently available drugs for the treatment of type 2 diabetes mellitus could also be used to treat glucocorticoid induced hyperglycaemia, but there are only a few published studies regarding the efficacy of these agents in glucocorticoid induced hyperglycaemia. However, few key principles should be employed in choosing glucose lowering drugs in patients. Metformin could be considered as one of the agents of choice in these patients, as it increases insulin sensitivity and counteracts the chief mechanism of pathogenesis of glucocorticoid induced hyperglycaemia. However, as some of the patients on long term glucocorticoids are either hypoxic or have renal insufficiency, metformin may not be the suitable option for them due to increased risk of lactic acidosis. Long acting sulfonylureas were first used in renal transplant patients with achievement of good blood glucose control in about $25 \%$ of the patients (2). The relatively low cost and prompt insulin secretory action are some of their benefits. The presence of drugs that are predominantly excreted by the kidneys (e.g. gliclazide) or drugs metabolised mainly by the liver (e.g. glypizide) are advantageous in advanced liver or renal failure. However, long acting sulfonylureas may increase the risk of hypoglycaemia, especially in patients with deteriorating renal function (13). Thiazolidinediones (glitazones) are also a treatment option. They have been used successfully in post transplant diabetes mellitus patients, either alone or in combination with other oral agents $(14,15)$. However there is increasing concern regarding oedema, congestive cardiac failure and risk of cardiovascular disease with their use (16). This may be detrimental for patients on glucocorticoids, which by themselves are associated with other metabolic complications like hyperlipidaemia, that have an additional detrimental effect on co-exisitng compromised renal function, which by itself is a risk factor for cardiac disease. There is emerging evidence of increasing fracture risk for women on long term glitazones (17), and with the increased risk of osteoporosis with glucocorticoid use, the combined risks would actually preclude their use in this specific group of patients.

In view of high doses of steroids being required to induce a therapeutic response in most diseases, they generally induce very severe insulin resistance, which may not be controlled adequately with non insulin therapy (11). Therefore insulin treatment would be necessary in most patients with glucocorticoid induced hyperglycaemia, except for the few who are on small doses of steroids. Insulin can be used safely and effectively in most patients. There are several options for treatment such as using basal insulin, prandial insulin or a pre mixed regime. There is a paucity of randomised clinical trials on the best insulin regime for patients with glucocorticoid induced hyperglycaemia. However, a recently concluded study comparing prandial, basal and pre mixed insulin in type 2 diabetes mellitus demonstrated that each regime reduces the HbA1C values significantly, but the blood sugar control was slightly better with prandial or pre mixed insulin regimes (18). These results could possibly be extrapolated to the steroid induced hyperglycaemia patients. But prandial and pre mixed insulin regimes have the disadvantage of being multiple, and complex which can reduce patient compliance with a higher risk of hypoglycaemia (1).

As patients treated with once daily glucocorticoids typically have normal fasting glucose levels with elevated post prandial values, a prandial insulin regime that is calculated according to the patient's body weight, meal pattern and caloric consumption, has been used as one appropriate strategy (11). Basal insulin would only be added to those on higher doses or multiple daily doses of glucocorticoids, or to those with pre-existing diabetes. However, frequent insulin injections, especially for patients who are already burdened by another chronic illness may actually reduce compliance. It can also be argued that the normal fasting blood glucose levels with gradual elevation of blood glucose throughout the day, resulting in post prandial hyperglycaemia, is related to the time course of insulin resistance induced by glucocorticoids rather than due to a specific defect in post prandial insulin secretion (2). In pregnancy, which is also a state of insulin resistance, the requirement of basal insulin increases with advancing pregnancy but the prandial insulin requirement remain stable (2). 
Therefore the best approach to ensure patient compliance and adequate control of blood glucose is basal insulin in the morning, calculated according to the body weight and the glucorticoid dose (1). Since isophane insulin has a peak action after 4 - 8 hours of administration with a duration of action for 12 to 16 hours, which mirrors the peak and duration of action of the prednisolone, the preference would be to use isophane insulin as the basal insulin type. The substitution of insulin glargine or determir would be appropriate when dexamethasone is used as it has even longer hyperglycaemic effects (2). Prandial insulin could be added if blood glucose fails to be controlled with the above regime, or, if the patient has preexisting diabetes. Patient compliance with this treatment would be better, as it involves just a single dose of insulin in the morning and the patient can adjust the dose according to his/her steroid intake.

\section{Conclusion}

Glucocorticoids are the treatment of choice for numerous conditions, often used long term, with a clear therapeutic benefit. However, it is often associated with a number of adverse events, with glucocorticoid induced insulin resistance leading to hyperglycaemia being a common problem. It is important to treat this hyperglycaemia as if uncontrolled is associated with acute inflammation and endothelial dysfunction. Glucocorticoid induced hyperglycaemia can be treated with either oral hypoglycaemic agents or insulin, but insulin is the more suitable treatment option.

\section{References}

1. Gulliford MC, Charlton J, Latinovic R. Risk of diabetes associated with prescribed glucocorticoids in a large population. Diabetes Care 2006; 29: 2728 - 29.

2. John N, Thurby L. Glucocorticoid induced hyperglycemia. Endocrine Practice 2009; 15.

3. Mokshagundam SP, Peiris AN. Drug induced disorders of glucose metabolism. Leahy JL, Clark NG, Cefalu WT, editors. Medical Management of Diabetes Mellitus. New York: Marcel Decker; 2000. P 201 - 15.

4. Uzu T, Harada T, Sakaguchi M. Glucocorticoid induced diabetes mellitus: prevalence and risk factors. Nephron Clin Pract 2007; 105: c54-c57.

5. Iwamoto T, Kagawa Y, Naito Y, Kuzuhara S, Kojima M. Steroid-induced diabetes mellitus and related risk factors in patients with neurologic diseases. Pharmacotherapy 2004; 24: 508 - 14 .
6. Wajngot A, Giacca A, Grill V, et al. The diabetogenic effects of glucocorticoids are more pronounced in low than high insulin responders. Proc Natl Acad Sci USA 1992; 89: $6035-9$.

7. Matsumoto K, Yamasaki H, Akazawa S, et al. High dose but not low dose dexamethasone impairs glucose tolerance by inducing compensatory failure of pancreatic beta-cells in normal men. J clin Endocrinol Metabol 1996; 81: 2621 - 6.

8. Vincenti F, Friman S, Scheuermann E, et al. Results of an international, randomized trial comparing glucose metabolism disorders and outcome with cyclosporine versus tacrolimus. Am J Transplant 2007; 7: 1506 - 14.

9. Shah P. Glucocorticoid induced diabetes mellitus. Retrieved from http://www.mayoclinic.org/medicalprofs/gluco corticoid-induced-diabetes.html

10. Gustavson SM, Sandoval DA, Ertl AC, Bao S, Raj SR, Davis SN. Stimulation of both type I and type II corticosteroid receptors blunts counter regulatory responses to subsequent hypoglycemia in healthy man. Am J Physiol Endocrinol Metab 2008; 294: E506-E512.

11. Trence DL, Management of patients on chronic glucocorticoid therapy: an endocrine perspective. Prim care 2003; 30: $593-605$.

12. Ceriello A, Esposito K, Piconi L, et al. Oscillating glucose is more deleterious to endothelial function and oxidative stress than mean glucose in normal and type 2 diabetic patients. Diabetes 2008; 57: 1349 - 54.

13. Roy D, Michael F. New-onset diabetes mellitus in the kidney recipient: diagnosis and management strategies. Clin J Am Soc Nephrol 2008; 3: S38-S48.

14. Luther P, Balswin D Jr. Pioglitazone in the management of diabetes mellitus after transplantation. Am J Transplant 2004; 2135 - 8.

15. Villanueva G, Baldwin D. Rosiglitazone therapy of post transplant diabetes mellitus. Transplantation 2005; 80: $1402-5$.

16. Largo RM, Singh PP, Nesto RW. Congestive heart failure and cardiovascular death in patients with prediabetes and type 2 diabetes given thiazolidinendiones: a meta-analysis of randomised clinical trials. Lancet 2007; 370: 1129-36.

17. Yoon K, Singh S, Furberg C. Long term use of thiazolidinediones and fractures in type 2diabetes: a metaanalysis. CMAJ 2009; 180(1): 32 - 39.

18. Holman R, Kerensa I, Farmer A. Addition of biphasic, prandial, or basal insulin to oral therapy in type 2 diabetes. N Engl J Med 2007; 357: 1716- 30. 